\title{
EFECTO DE DIFERENTES FUENTES DE NITRÓGENO EN EL CONTENIDO DE CAROTENOIDES Y CLOROFILA DE CUATRO CEPAS PERUANAS DE Dunaliella salina TEOD.
}

\section{EFFECT OF DIFFERENT NITROGEN SOURCES ON THE CAROTENOID AND CHLOROPHYLL CONTENT OF FOUR PERUVIAN STRAINS OF Dunaliella salina TEOD.}

\author{
Román Felipe Serpa Ibáñez y Abelardo Calderón Rodríguez ${ }^{1}$
}

Resumen

Se estudió el efecto de diferentes fuentes de nitrógeno $\left(\mathrm{NO}_{3} \mathrm{NH}_{4}, \mathrm{SO}_{4}\left(\mathrm{NH}_{4}\right)_{2}, \mathrm{NO}_{3} \mathrm{~K}, \mathrm{NO}_{3} \mathrm{Na}\right.$ y Urea) en el crecimiento y contenido de pigmentos carotenoides y clorofila en cuatro cepas peruanas de Dunaliella salina. A los 27 días, el sulfato de amonio (y después el nitrato de amonio) produjeron la más alta densidad celular y contenido de pigmentos (mg/l) en las siguientes cepas: FV-002 (Salina de Huacho) y FV-003 (Salina de Chilca). Sin embargo, en los tratamientos con sulfato de amonio, se observó el blanqueamiento de los cultivos algunos días después.

Palabras claves: carotenoides, clorofilas, densidad celular, Dunaliella

\begin{abstract}
The effect of different nitrogen sources $\left(\mathrm{NO}_{3} \mathrm{NH}_{4}, \mathrm{SO}_{4}\left(\mathrm{NH}_{4}\right)_{2}, \mathrm{NO}_{3} \mathrm{~K}, \mathrm{NO}_{3} \mathrm{Na}\right.$ and Urea) on the growth and pigment content (carotenoids and chlorophylls) of four Peruvian strains of Dunaliella salina was studied. At 27 days of age, ammonium sulfate (and after that, ammonium nitrate) produced the highest cellular density and pigment content $(\mathrm{mg} / \mathrm{l})$ in the following strains: FV-002 (Salina de Huacho) y FV-003 (Salina de Chilca). Nevertheless, in the ammonium sulfate treatments, bleaching of the cells was evident some days later.
\end{abstract}

Key words: carotenoids, chlorophylls, cellular density, Dunaliella

\section{Introducción}

Uno de los nutrientes más importantes en el crecimiento de un alga, es el nitrógeno. Algunos investigadores creen que la mejor fuente de nitrógeno para el crecimiento de Dunaliella salina es el $\mathrm{NO}_{3} \mathrm{Na}$ y que fuentes de nitrógeno como las sales de amonio (acetato de amonio, nitrato de amonio, sulfato de amonio y cloruro de amonio) son fuentes menos efectivas, además esas sales de amonio a concentraciones superiores a $2.5 \mathrm{M}$ pueden ser letales. Pero esas sales de amonio a bajas concentraciones en medios con buffer; estimulan el crecimiento comparado con los nitratos. Así, la reducción de nitrato requiere energía y si la energía suplementada es limitada, el mayor crecimiento ocurrirá con el amonio. También existen fuentes de nitrógeno orgánicas tales como la urea, pero generalmente son menos eficientes que las fuentes de nitrógeno inorgánicas (Barbarena et al., 1990; Ben-Amotz et al., 1982). En el presente estudio se evaluó la influencia de diferentes fuentes de nitrógeno en la producción de $\beta$-caroteno y clorofila en condiciones de laboratorio en cuatro cepas silvestres de Dunaliella salina Teod. colectados en las salinas de Huacho, Chilca, Otuma y Los Chimus en la costa Peruana.

\section{Materiales y Métodos}

Se usaron cuatro cepas de Dunaliella salina de distinto origen geográfico: cepa de las Salinas de Los Chimus (FV-001), cepa de las Salinas de Huacho (FV002), cepa de las Salinas de Chilca (FV-003) y cepa de las Salinas de Otuma (FV-004).

Estas fueron cultivadas en medio enriquecido (Tabla 1). Se probaron cinco diferentes fuentes de nitrógeno (nitrato de sodio, nitrato de potasio, nitrato de amonio, sulfato de amonio y urea) versus cuatro cepas de Dunaliella salina.

La concentración de CINa usada en las pruebas fue de 3.0 M. Respecto a las concentraciones de las fuentes de Nitrógeno en todos los casos se usó 5 $\mathrm{mM} / 1$. La concentración del fosfato fue de $0.2 \mathrm{mM} / 1$, teniendo en cuenta que el $\mathrm{PO}_{4} \mathrm{HNa}_{2}$ se usó solo con el $\mathrm{NO}_{3} \mathrm{Na}$, y en todos los demás se usó el $\mathrm{PO}_{4} \mathrm{HK}_{2}$; la concentración de micronutrientes fue aquella que se usa en el medio Guillard, con una variación en la concentración del $\mathrm{H}_{3} \mathrm{BO}_{3}$ (Tabla 1).

En cada tratamiento se usó $150 \mathrm{ml}$. de la solución experimental y se inoculó con $15 \mathrm{ml}$ del cultivo stock mantenido en el mismo medio en fase estacionaria temprana. Cada tratamiento fue repetido tres veces en diferentes lapsos de tiempo $(1 \mathrm{x}, 2 \mathrm{x}$ y $3 \mathrm{x})$. Los tratamientos se expusieron a un fotoperíodo de 12:12 (L: O) y fueron mantenidos a una densidad de flujo 
fotónico de aproximadamente $272 \mu \mathrm{E} / \mathrm{m}^{2} . \mathrm{s}, \quad \mathrm{y}$ temperatura semicontrolada, con agitación en cada muestreo cada tres días durante 27 días.

Tabla 1. Medio de Cultivo para Dunaliella salina.

\begin{tabular}{|c|c|c|c|}
\hline \multicolumn{2}{|c|}{ Macronutrientes } & \multicolumn{2}{|c|}{ Micronutrientes } \\
\hline $\mathrm{Na} \mathrm{Cl}(3 \mathrm{M})$ & $175.32 \mathrm{~g} / 1$ & Na EDTA & $4.36 \mathrm{mg} / \mathrm{l}$ \\
\hline $\begin{array}{l}\text { Fuente de } \\
\text { Nitrógeno }\end{array}$ & $5 \mathrm{mM} / 1 \mathrm{de}$ & $\mathrm{FeCl}_{3}$ & $3.15 \mathrm{mg} / \mathrm{l}$ \\
\hline $\begin{array}{l}\mathrm{KH}_{2} \mathrm{PO}_{4} \quad \text { o } \\
\mathrm{NaH}_{2} \mathrm{PO}_{4}\end{array}$ & $0.2 \mathrm{mM} / 1$ & $\mathrm{Cl}_{2} \mathrm{Mn} \cdot 2 \mathrm{H}_{2} \mathrm{O}$ & $180 \mu \mathrm{g} / \mathrm{l}$ \\
\hline & & $\mathrm{SO}_{4} \mathrm{Cu} .5 \mathrm{H}_{2} \mathrm{O}$ & $9.8 \mu \mathrm{g} / 1$ \\
\hline & & $\mathrm{Cl}_{2} \mathrm{Co} . \mathrm{H} 2 \mathrm{O}$ & $10.5 \mu \mathrm{g} / 1$ \\
\hline & & $\mathrm{Na}_{2} \mathrm{MoO}_{4} \cdot \mathrm{H}_{2} \mathrm{O}$ & $6.3 \mu \mathrm{g} / \mathrm{l}$ \\
\hline & & $\mathrm{SO}_{4} \mathrm{Zn} \cdot \mathrm{H}_{2} \mathrm{O}$ & $22 \mu \mathrm{g} / 1$ \\
\hline & & $\mathrm{H}_{3} \mathrm{BO}_{3}$ * & $4.45 \mu \mathrm{g} / 1$ \\
\hline
\end{tabular}

Durante el crecimiento los cultivos fueron periódicamente muestreados y analizadas para determinar la densidad celular y la concentración de pigmentos. Para determinar la densidad celular cada muestra fue fijada con lugol. Los conteos se hicieron empleando una cámara Neubauer standard de $0.1 \mathrm{~mm}$ de altura y $1 \mathrm{~mm}^{2}$ de área. Las curvas de crecimiento se ajustan a la ecuación $\mathrm{N}=\mathrm{N}_{\mathrm{O}}$ $2 \mathrm{~T} /$ td donde $\mathrm{N}_{\mathrm{O}}$ es la densidad celular inicial, $\mathrm{T}$ es el tiempo de evaluación y td es la tasa de duplicación celular.

Para llevar a cabo la extracción de los pigmentos se tomaron alícuotas del cultivo y se dispusieron en tubos de centrifuga de plástico. Se centrifugó a $5000 \mathrm{~g}$ por una hora. El pellet fue lavado directamente con acetona pura $(\sim 3.2 \mathrm{ml})$, y agitado en vortex posteriormente. Se agregó $0.8 \mathrm{ml}$ de agua destilada con el fin de llegar a la concentración de acetona de 80 $\% \mathrm{v} / \mathrm{v}$.

La concentración de carotenoides totales $(\beta-$ caroteno como componente con la mayor concentración) fue cuantificada usando $\mathrm{E}_{1 \%}{ }^{480} \mathrm{~nm}$ (coeficiente de extinción) de 2273 a $480 \mathrm{~nm}$. La concentración de clorofila en $\mathrm{mg} / \mathrm{l}$ fue cuantificada como lo describe Arnon (1949).

Con la finalidad de determinar diferencias significativas en la densidad celular, en el contenido de clorofilas y en los carotenos provocados por las diferentes fuentes de Nitrógeno en las cepas, se hizo una prueba de análisis de variancia con arreglo factorial y además se hizo las pruebas de comparación de Tuckey.

\section{Resultados}

Efecto de diferentes fuentes de nitrógeno sobre la densidad celular.

En todas las cepas, las mayores densidades finales se produjeron en los medios que tuvieron $\mathrm{SO}_{4}\left(\mathrm{NH}_{4}\right)_{2}$ (Tabla 2, Figura 6) con una alta diferencia significativa. Fue seguida por el $\mathrm{NO}_{3} \mathrm{NH}_{4}$, y luego por los nitratos que produjeron densidades finales muy similares. Con respecto a las densidades celulares producidas en cada cepa en su totalidad, resaltó la cepa de Huacho que tuvo la mayor densidad celular promedio.

Considerando todo el intervalo del cultivo, el $\mathrm{SO}_{4}\left(\mathrm{NH}_{4}\right)_{2}$ siempre produjo densidades celulares promedio superiores a los medios con $\mathrm{NO}_{3} \mathrm{NH}_{4}$ en las cepas de Huacho, Chilca y Otuma. Por el contrario, las menores densidades se produjeron en los tratamientos con Urea (tabla 2). Las cepas de Los Chimus, Chilca y Huacho siempre tuvieron densidades celulares finales mayores a $0.5 \times 10^{5}$ células $/ \mathrm{ml}$ en las fuentes de nitrógeno analizadas (tabla 2).

En las cepas de Los Chimus a pesar que durante todo el intervalo de cultivo, el medio con $\mathrm{NO}_{3} \mathrm{NH}_{4}$ alcanzó mayores densidades, la mayor densidad final se produjo en el medio con el $\mathrm{SO}_{4}\left(\mathrm{NH}_{4}\right)_{2}$. Cabe destacar que los medios con $\mathrm{NO}_{3} \mathrm{~K}$ y $\mathrm{NO}_{3} \mathrm{Na}$ en los primeros quince días de cultivo tuvieron mayor densidad que el $\mathrm{SO}_{4}\left(\mathrm{NH}_{4}\right)_{2}$ (Figura 2). En esta cepa, el tratamiento que alcanzó la mayor tasa de crecimiento fue el $\mathrm{NO}_{3} \mathrm{Na}$. 27 de cultivo.

\begin{tabular}{lcccccc}
\hline Cepas & pr/ $/ \mathrm{NO}_{3} \mathrm{~K}$ & $\mathrm{pr} / \mathrm{NO}_{3} \mathrm{Na}$ & $\mathrm{pr} / \mathrm{NO}_{3} \mathrm{NH}_{4}$ & $\mathrm{pr} / \mathrm{SO}_{4}\left(\mathrm{NH}_{4}\right)_{2}$ & pr/urea & promedio \\
\hline Los Chimus & 119433 & 127200 & 227200 & 237767 & 109333 & 164186.6 \\
Chilca & 177233 & 141100 & 352800 & 395033 & 136100 & 240453.2 \\
Huacho & 198867 & 223333 & 341667 & 431633 & 144433 & 267986.6 \\
Otuma & 21133 & 21100 & 87400 & 125000 & 28333 & 56593.2 \\
Promedio & 129166.5 & 128183.3 & 252267 & 297358.25 & 104550 & 182304.9 \\
\hline
\end{tabular}

as : *

En la cepa de Huacho los tratamientos con $\mathrm{NO}_{3} \mathrm{~K}$ y el $\mathrm{NO}_{3} \mathrm{Na}$ tuvieron densidades finales y tasas de crecimiento sin diferencia significativa (Figura 1 en Apéndice, Tabla 2)

En la cepa de Chilca, el medio con $\mathrm{SO}_{4}\left(\mathrm{NH}_{4}\right)_{2}$ alcanzó una densidad celular final semejante a la cepa de Huacho $\left(\sim 3.5 \times 10^{5}\right.$ células $\left./ \mathrm{ml}\right)$, además tuvo una tasa de crecimiento sin diferencia significativa con la tasa producida en el tratamiento con $\mathrm{NO}_{3} \mathrm{NH}_{4}$ (Tabla 2, Figura 1).

Finalmente en la cepa de Otuma la mayor densidad celular $\left(<1.25 \times 10^{5}\right.$ células $\left./ \mathrm{ml}\right)$ se dio en el tratamiento con $\mathrm{SO}_{4}\left(\mathrm{NH}_{4}\right)_{2}$, pero fue a su vez la menor densidad celular alcanzada para ese tratamiento en las cuatro cepas. Los demás tratamientos tuvieron las menores densidades celulares producidas en las cuatro cepas $\left(<0.3 \times 10^{5}\right.$ células $\left./ \mathrm{ml}\right)$ (Figura 1, Tabla 2).

Si comparamos las tasas de crecimiento de las cepas con respecto a cada nutriente podremos notar que hubo por lo menos de 2 a 3 cepas sin diferencias significativas en sus valores. Sin embargo, las tasas de 
crecimiento en el $\mathrm{NO}_{3} \mathrm{NH}_{4}$ no expresaron diferencias significativas entre las cepas.

Efecto de diferentes fuentes de nitrógeno sobre el contenido de carotenoides totales en $\mathrm{mg} / \mathrm{l}$

Las mayores concentraciones de carotenoides totales se dieron en los tratamientos con $\mathrm{SO}_{4}\left(\mathrm{NH}_{4}\right)_{2}$ y $\mathrm{NO}_{3} \mathrm{NH}_{4}$. En las cepas de Huacho, Chilca y Otuma, el medio con $\mathrm{SO}_{4}\left(\mathrm{NH}_{4}\right)_{2}$ fue el que obtuvo en promedio, las mayores concentraciones de carotenoides. En la cepa de Los Chimus, fue el tratamiento con $\mathrm{NO}_{3} \mathrm{NH}_{4}$ el que dió la mayor concentración promedio (Tabla 3, Figura 2).

Las cepas de Los Chimus, Huacho y Chilca tuvieron las menores concentraciones de carotenoides finales promedio en el medio con Urea. Considerando las cuatro cepas, la cepa de Otuma cultivada en Urea, fue la que tuvo la menor concentración de carotenoides. La mínima concentración de carotenoides en la cepa de Otuma se produjo en el tratamiento con $\mathrm{NO}_{3} \mathrm{~K}$ y $\mathrm{NO}_{3} \mathrm{Na}$ (Tabla 3).

Tabla 3. concentracion de carotenoides totales(mg/l).

\begin{tabular}{lcccccc}
\hline Cepas & pr/ $/ \mathrm{NO}_{3} \mathrm{~K}$ & $\mathrm{pr} / \mathrm{NO}_{3} \mathrm{Na}$ & $\mathrm{pr} / \mathrm{NO}_{3} \mathrm{NH}_{4}$ & $\mathrm{pr} / \mathrm{SO}_{4}\left(\mathrm{NH}_{4}\right)_{2}$ & pr/urea & Promedio \\
\hline Los & 0.7393 & 0.9739 & 1.6107 & 1.3934 & 0.5861 & 1.06068 \\
Chimus & & 0.9152 & 1.8063 & 2.3129 & 0.5051 & 1.06068 \\
Chilca & 1.0277 & 1.4166 & 1.5019 & 1.8639 & 0.5379 & 1.06068 \\
Huacho & 0.9112 & 1.014 & 1.3718 & 0.2366 & 1.06068 \\
Otuma & 0.1495 & 0.1642 & 1.014 & \\
promedio & 0.706925 & 0.86748 & 1.483225 & 1.7355 & 0.466425 & 1.06068 \\
\hline
\end{tabular}

Todas las comparaciones : *

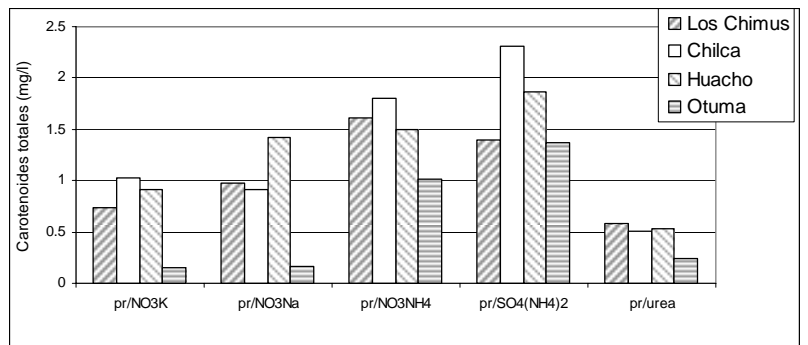

Figura 2. Concentración de Carotenoides totales en $\mathrm{mg} / \mathrm{l}$ de cuatro cepas de Dunaliella salina al día 27 por efecto de diferentes fuentes de Nitrógeno en los medios de cultivo

Tabla 3. Concentración de carotenoides totales(mg/l) al día 27 de cultivo

\begin{tabular}{lcccccc}
\hline Cepas & pr/ $/ \mathrm{NO}_{3} \mathrm{~K}$ & $\mathrm{pr} / \mathrm{NO}_{3} \mathrm{Na}$ & $\mathrm{pr} / \mathrm{NO}_{3} \mathrm{NH}_{4}$ & $\mathrm{pr} / \mathrm{SO}_{4}\left(\mathrm{NH}_{4}\right)_{2}$ & pr/urea & promedio \\
\hline Los & 0.7393 & 0.9739 & 1.6107 & 1.3934 & 0.5861 & 1.06068 \\
Chimus & .0277 & 0.9152 & 1.8063 & 2.3129 & 0.5051 & 1.06068 \\
Chilca & 1.029 & 1.8639 & 0.5379 & 1.06068 \\
Huacho & 0.9112 & 1.4166 & 1.5019 & 1.3718 & 0.2366 & 1.06068 \\
Otuma & 0.1495 & 0.1642 & 1.014 & 1.3756 \\
promedio & 0.706925 & 0.86748 & 1.483225 & 1.7355 & 0.466425 & 1.06068 \\
\hline
\end{tabular}

Todas las comparaciones : *
Con respecto al efecto de la temperatura, cada cepa en los diferentes tratamientos responde de forma particular. Así, en las cepas de Chilca y Huacho, las temperaturas inferiores a $24{ }^{\circ} \mathrm{C}$ en los medios con sales de amonio, produjeron las mayores concentraciones de carotenoides; sin embargo en los medios con nitrato o urea las temperaturas entre $27 \mathrm{y}$ $28{ }^{\circ} \mathrm{C}$ produjeron las mayores concentraciones de carotenoides (Tabla 3).

La cepa de Los Chimus presenta el comportamiento inverso en las sales de amonio, nitrato y urea. Es decir con temperaturas entre 27 y 28 ${ }^{\circ} \mathrm{C}$ en los medios con sales de amonio se produjeron las mayores concentraciones de carotenoides, en cambio en los medios con nitrato a temperaturas inferiores a $24{ }^{\circ} \mathrm{C}$ se produjeron las mayores concentraciones de carotenoides.

Es notorio que cada cepa responde de una manera diferente con cada tratamiento. En los medios con $\mathrm{NO}_{3} \mathrm{NH}_{4} \quad$ ó $\quad \mathrm{SO}_{4}\left(\mathrm{NH}_{4}\right)_{2}$ ó $\mathrm{NO}_{3} \mathrm{~K}$ las mayores concentraciones promedio de carotenoides se produjeron en la cepa de Chilca. En el medio con el $\mathrm{NO}_{3} \mathrm{Na}$ la mayor concentración promedio se produjo en la cepa de Huacho. En el medio con Urea la mayor concentración se dio en la cepa de Los Chimus (Tabla 3).

Las concentraciones de carotenoides totales promedio fueron siempre mayores a $3.5 \mathrm{pg} /$ célula al final del cultivo (Figura 4). Pero fue en la cepa de Otuma que se produjeron las mayores concentraciones de carotenoides promedio (mayor a 7 pg/célula) considerando todos los medios de cultivo. Solo en las cepas de Chilca y Huacho, el tratamiento con $\mathrm{SO}_{4}\left(\mathrm{NH}_{4}\right)_{2}$ obtuvo las mayores concentraciones (esto se correlaciona con las concentraciones de carotenoides expresadas en $\mathrm{mg} / \mathrm{l}$ ). Pero en la cepa de Los Chimus la mayor concentración promedio se produjo en el tratamiento con $\mathrm{NO}_{3} \mathrm{Na}$; y en la cepa de Otuma se produjo en el tratamiento con Urea (Tabla 3.1, Figura 4).

Efecto de diferentes fuentes de nitrógeno sobre el contenido de clorofila en $\mathrm{mg} / \mathrm{l}$

Hay una alta correlación de la concentración de la clorofila con la concentración de carotenoides producidas por los medios con $\mathrm{NO}_{3} \mathrm{NH}_{4}$ en las cuatro cepas (Tabla 3 y 4 ). En las cuatro cepas las concentraciones de clorofila fueron menores a las concentraciones de carotenoides en los medios con $\mathrm{NO}_{3} \mathrm{~K}, \mathrm{NO}_{3} \mathrm{Na}$ y $\mathrm{NO}_{3} \mathrm{NH}_{4}$ a excepción de la concentración de clorofila presente en el medio con $\mathrm{NO}_{3} \mathrm{Na}$ en la cepa de Otuma (Tabla 4, Figura 3).

En los medios con $\mathrm{SO}_{4}\left(\mathrm{NH}_{4}\right)_{2}$, las cepas de Chilca, Otuma y Huacho alcanzaron concentraciones de clorofila mayores a la de 
carotenoides a temperaturas entre 27 y $28^{\circ} \mathrm{C}$. En el caso de la Urea, en la cepa de Los Chimus la concentración de clorofila fue mayor a la de carotenoides a temperaturas inferiores a $24^{\circ} \mathrm{C}$. Pero en la cepa de Huacho se produjo este hecho a temperaturas entre 27 y $28^{\circ} \mathrm{C}$ (Tablas 3 y 4 ).

Figura 3.1. Concentración de carotenoides totales (pg/celúla) al día 27 de cultivo.

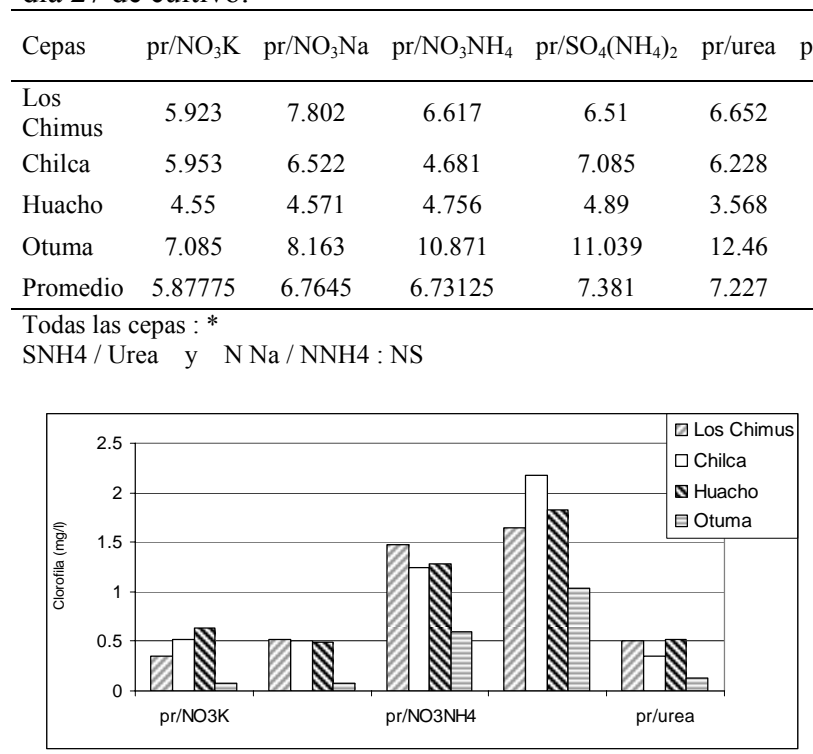

Figura 3. Concentración de Clorofila en $\mathrm{mg} / \mathrm{l}$ de cuatro cepas de Dunaliella salina al día 27 por efecto de diferentes fuentes de Nitrógeno en los medios de cultivo.

Tabla 4. Concentración de clorofila (mg/l) al día 27 de cultivo.

\begin{tabular}{|c|c|c|c|c|c|c|}
\hline Cepas & $\mathrm{pr} / \mathrm{NO}_{3} \mathrm{~K}$ & $\mathrm{pr} / \mathrm{NO}_{3} \mathrm{Na}$ & $\mathrm{pr} / \mathrm{NO}_{3} \mathrm{NH}_{4}$ & $\mathrm{pr} / \mathrm{SO}_{4}\left(\mathrm{NH}_{4}\right)_{2}$ & $\mathrm{pr} /$ urea & Promedio \\
\hline $\begin{array}{l}\text { Los } \\
\text { Chimus }\end{array}$ & 0.3509 & 0.5192 & 1.4789 & 1.6492 & 0.5102 & 0.90168 \\
\hline Chilca & 0.5219 & 0.4996 & 1.2434 & 2.1753 & 0.3439 & 0.95682 \\
\hline Huacho & 0.6307 & 0.4931 & 1.2822 & 1.8319 & 0.5213 & 0.95184 \\
\hline Otuma & 0.0802 & 0.0756 & 0.5937 & 1.0383 & 0.1354 & 0.38464 \\
\hline Promedio & 0.395925 & 0.39688 & 1.14955 & 1.673675 & 0.3777 & 0.79875 \\
\hline
\end{tabular}

NK - Na -Urea : NS

Chilca - Huacho : NS

Diferencias significativas : *

Diferencias no significativas : NS

Tabla 4.1. Concentración de clorofila (pg/célula) al día 27 de cultivo.

\begin{tabular}{|c|c|c|c|c|c|c|}
\hline Cepas & $\mathrm{pr} / \mathrm{NO}_{3} \mathrm{~K}$ & $\mathrm{pr} / \mathrm{NO}_{3} \mathrm{Na}$ & $\mathrm{pr} / \mathrm{NO}_{3} \mathrm{NH}_{4}$ & $\mathrm{pr} / \mathrm{SO}_{4}\left(\mathrm{NH}_{4}\right)_{2}$ & pr/urea & Promedio \\
\hline $\begin{array}{l}\text { Los } \\
\text { Chimus }\end{array}$ & 1.458 & 1.558 & 0.788 & 1.518 & 0.917 & 1.2478 \\
\hline Chilca & 1.375 & 1.297 & 1.053 & 1.906 & 0.873 & 1.3008 \\
\hline Huacho & 1.021 & 1.28 & 0.816 & 1.424 & 0.976 & 1.1034 \\
\hline Otuma & 1.58 & 1.159 & 1.108 & 1.545 & 1.176 & 1.3136 \\
\hline Promedio & 1.3585 & 1.3235 & 0.94125 & 1.59825 & 0.9855 & 1.2414 \\
\hline
\end{tabular}

$\mathrm{NK}-\mathrm{N} \mathrm{Na}: \mathrm{NS}$

Urea - N NH4 : NS

Chilca - Otuma y Chilca - Chimus :NS

La cepa de Los Chimus que se expuso al medio con $\mathrm{SO}_{4}\left(\mathrm{NH}_{4}\right)_{2}$ presentó concentraciones de clorofila menores a la de carotenoides. Este hecho también sucedió en la cepa de Chilca expuesta al medio con Urea (Tabla 3 y 4 ).

Cuando se comparó las concentraciones de clorofila en mg/l en el día 27 (día final de cultivo) en los medios con $\mathrm{NO}_{3} \mathrm{~K}, \mathrm{NO}_{3} \mathrm{Na}$ y Urea, no hubo diferencias significativas. Sucedió lo mismo cuando se comparó las concentraciones de clorofila en $\mathrm{mg} / \mathrm{l}$ en las cepas de Chilca y Huacho $(\alpha=$ $0.01)$

En todos los casos la concentración de clorofila expresada en $\mathrm{pg}$ /célula es menor que la concentración de carotenoides, además si establecemos una relación peso-peso $(\mathrm{g} / \mathrm{g})$ entre ambos podemos decir que la concentración de carotenoides es siempre mayor al doble de la concentración de clorofila en todas las unidades muéstrales. Cabe destacar que la máxima concentración de clorofila en $\mathrm{pg} / \mathrm{célula}$ reportada en el experimento (2.7969 pg/célula) se presentó cuando se cultivó a temperaturas cercanas a $30^{\circ} \mathrm{C}$ (Tabla 4.1 , Figura 5 ).

\section{Discusión}

Efecto de diferentes fuentes de nitrógeno sobre la densidad celular

Basándose en el primer análisis, se observa que, los medios de cultivo con sales de amonio produjeron las más altas densidades celulares en todas las cepas analizadas. En contraste, los medios con urea produjeron las menores densidades celulares.

Una de las razones de este comportamiento lo fundamenta Huppe \& Turpin (1994) que expresan que la asimilación del amonio es energéticamente menos costosa. En la asimilación del $\mathrm{NO}_{3}{ }^{-}$se requiere más energía y poder reductor, además de movilizar mas carbono que ocasiona tasas de liberación de $\mathrm{CO}_{2}$ relativamente altas por respiración.

Las cepas expresaron diferentes densidades celulares ante cada fuente de nitrógeno, pero fue la cepa de Huacho, la mas eficiente significativamente (mayor densidad celular) seguida en orden descendente por las cepas de Chilca, Los Chimus y Otuma. Lo que es claro es que cada cepa expresa un comportamiento particular como se ha observado en otras cepas de Dunaliella salina. (Giordano et al., 1994; Araneda et al., 1992 a; Araneda et al., 1992 b).

Cabe resaltar que el $\mathrm{SO}_{4}\left(\mathrm{NH}_{4}\right)_{2}$ expresa una mayor eficiencia en comparación al $\mathrm{NO}_{3} \mathrm{NH}_{4}$ considerando los 30 primeros días de cultivo. Es interesante resaltar que cuando se midió el $\mathrm{pH}$ en el medio con $\mathrm{SO}_{4}\left(\mathrm{NH}_{4}\right)_{2}$, el día 27, este fue menor a $7(\sim 6.7)$, en cambio en el medio con $\mathrm{NO}_{3} \mathrm{NH}_{4}$ el pH fue de $\sim 7.8$. Además días después el color del medio con $\mathrm{SO}_{4}\left(\mathrm{NH}_{4}\right)_{2}$ se perdió (proceso fotooxidativo). 


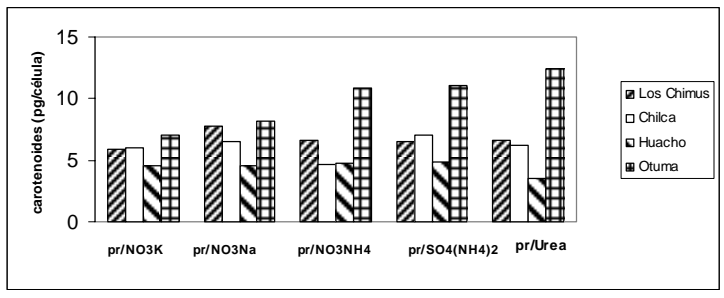

Figura 4. Concentración de Carotenoides en pg / Célula de cuatro cepas de Dunaliella salina al día 27 por efecto de diferentes fuentes de Nitrógeno en los medios de cultivo.

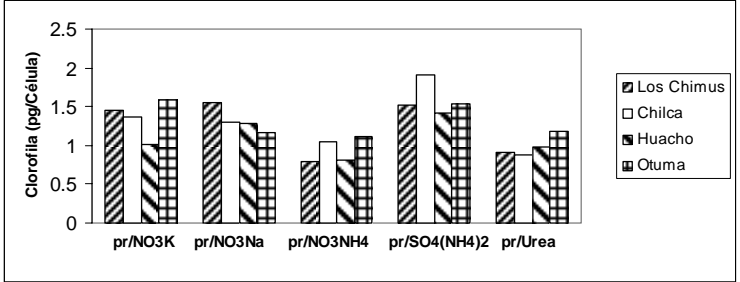

Figura 5. Concentración de Clorofila en pg / Célula de cuatro cepas de Dunaliella salina al día 27 por efecto de diferentes fuentes de Nitrógeno en los medios de cultivo.

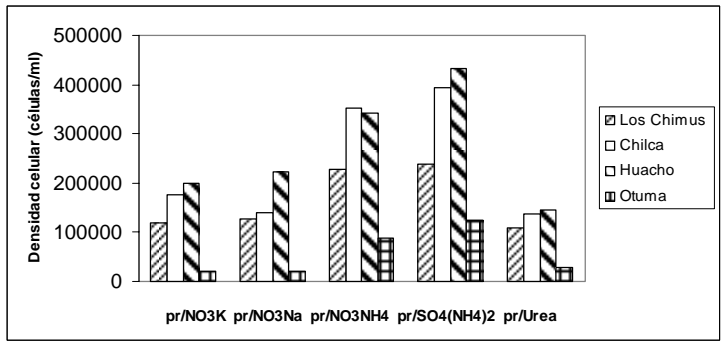

Figura 6. Densidad celular de cuatro cepas de Dunaliella salina por efecto de diferentes fuentes de Nitrógeno en los medios de cultivo.

La menor eficiencia (menor densidad final) del $\mathrm{NO}_{3} \mathrm{NH}_{4}$ parece indicar un cierto grado de inhibición del $\mathrm{NH}_{4}^{+}$en la asimilación del $\mathrm{NO}_{3}{ }^{-}$, que es una reacción que se da comúnmente en bacterias. Hubo en el medio con $\mathrm{NO}_{3} \mathrm{NH}_{4}$ un sistema amortiguador debido a la estrecha variación del $\mathrm{pH}$ en el medio, fenómeno que no ocurrió en el medio con $\mathrm{SO}_{4}\left(\mathrm{NH}_{4}\right)_{2}$ que promovió una acidificación del cultivo en la fase estacionaria. Esto último lo ha afirmado Ben Amotz (1989) además de haber mencionado que Dunaliella tiene un óptimo crecimiento en el rango de $\mathrm{pH}$ entre 7 a 9, y que pHs inferiores o superiores a este intervalo disminuyen la tasa de crecimiento aproximadamente a dos tercios.

Al menos en tres cepas (originadas de salinas costeras), la densidad celular final producida en el medio con $\mathrm{NO}_{3} \mathrm{Na}$ fue mayor al $\mathrm{NO}_{3} \mathrm{~K}$. Este mismo comportamiento se dio en la cepa chilena CONC-001 aislada de una salina costera. Dada estas evidencias podemos decir que las cepas aisladas de ecosistemas talasohalinos tienen una alta probabilidad de alcanzar densidades celulares más altas en medios con $\mathrm{NO}_{3} \mathrm{Na}$. Contrariamente la cepa de Chilca responde mejor al $\mathrm{NO}_{3} \mathrm{~K}$ al igual que algunas cepas chilenas de Dunaliella salina de ecosistemas atalasohalinos (CONC-003)(Araneda et al., 1992 a).

La poca eficiencia de la urea parece en principio estar relacionada a la baja actividad de la ureasa. Se sabe que esta enzima necesita níquel para su actividad y que su actividad enzimática puede ser inhibida por el buffer fosfato, el buffer tris o el ácido bórico (Mobley et al., 1995). Dado que en el presente experimento los medios tuvieron ácido bórico, este ácido pudo ser una de las causas del bajo crecimiento en los medios con urea. Otra posible razón es el comportamiento de la urea como solvente. La urea es un solvente caotrópico que tiende a desestabilizar la conformación de las proteínas (Wood, 1999). Se sabe que la Dunaliella salina no tiene pared celular, por lo tanto estaría más expuesta al efecto caotrópico de la urea.

Reay (1999), recientemente ha comprobado que las variaciones de temperatura tienen una gran influencia en el crecimiento del alga $D$. tertiolecta en medios con nitratos. Afirma que la afinidad específica de esta alga por las sales de nitrato es fuertemente influenciada por la temperatura. Por el contrario la temperatura tiene menos influencia en las sales de amonio y por tanto en las tasas de crecimiento producidas. Al parecer en el experimento las cepas de Chilca, Huacho y Otuma siguen este comportamiento. Efecto de diferentes fuentes de nitrógeno sobre el contenido de carotenoides

La acumulación de carotenoides y xantofilas es indicativa de un cierto grado de estrés provocado por un estimulo. Así, el grado de acumulación de estas sustancias en las algas es proporcional a la intensidad del estimulo estresante.

Los mayores contenidos de carotenoides se produjeron en los medios con sales de amonio en forma similar al efecto producido sobre las densidades celulares. Además, se ha observado que el contenido de carotenoides producido por efecto de cada fuente de nitrógeno tiene una alta correlación con la densidad celular.

El hecho de que en células como la Dunaliella, las fuentes de nitrógeno como el amonio no necesitan tanta energía en la asimilación como la que se usa en el nitrato, hace disponible energía que se puede direccionar a la síntesis de carotenoides (Huppe et al., 1994; Reay et al., 1999).

Cuando un alga como Dunaliella salina esta creciendo en medios con nitratos, el $\mathrm{pH}$ tiende a aumentar. Esta alcalinización promueve la precipitación de sales de fosfato o sulfato lo que puede ocasionar un estado de deficiencia. Ante esta última situación las células disminuyen la actividad del fotosistema II y por tanto de la fotosíntesis. Así el 
exceso de irradiación puede entonces ocasionar daños en los centros de reacción y, promover la acumulación de xantofilas (respuesta primaria) con un posible rol fotoprotector (Wycoff et al., 1998).

Muchos investigadores afirman que la exposición a una alta salinidad, alta intensidad luminosa, deficiencia de nitrógeno, temperaturas extremas $\mathrm{y}$ variaciones de $\mathrm{pH}$ estimulan una mayor producción de carotenoides. La mayor concentración de carotenoides en tres de cuatro cepas en los medios con $\mathrm{SO}_{4}\left(\mathrm{NH}_{4}\right)_{2}$ parece ser debido a la disminución de $\mathrm{pH}$ (menor a 7)en este nutriente. Así, la presencia del $\mathrm{SO}_{4}^{-}$ ocasionó un efecto carotenogénico en los medios con $\mathrm{SO}_{4}\left(\mathrm{NH}_{4}\right)_{2}$ que también coadyuvo a la disminución del $\mathrm{pH}$ ocasionado por la asimilación del amonio en las cepas de Chilca, Huacho y Otuma. Cabe destacar que en la cepa de Los Chimus, la mayor concentración se dio en el medio con nitrato de amonio. Esta mayor sensibilidad de la cepa de Los Chimus por el nitrato de amonio parece denotar que esta cepa es genotípicamente diferente a las cepas anteriormente mencionadas.

El hecho que la Urea provocara la mayor concentración de carotenoides (en mg/l y pg/célula) en la cepa de Otuma por encima de la concentración de carotenoides producidas por el $\mathrm{NO}_{3} \mathrm{~K}$ y $\quad \mathrm{NO}_{3} \mathrm{Na}$ denota que la actividad ureasa en esta cepa y su mayor densidad celular produjeron probablemente un gasto energético menor al gasto necesario para la producción celular y de carotenoides en los medios con nitratos. Este comportamiento aunado a la poca cantidad de carotenoides totales en $\mathrm{mg} / \mathrm{l}$ reportadas en esta cepa podría denotar ciertas características genotípicas distintivas. El análisis de los contenidos de carotenoides producidos en ambientes abiertos permitiría comprobar tal enunciado.

Los contenidos de carotenoides producidos en la cepa de Otuma durante los meses de verano ( $>$ a 30 $\left.{ }^{\circ} \mathrm{C}\right)$ corroboran el efecto inhibitorio de las temperaturas altas. Se sabe que temperaturas superiores o inferiores a las temperaturas óptimas estimulan la reducción de la CoQ (Coenzima Q). El aumento desmesurado de este componente estimula la producción de radicales oxidantes que pueden destruir la estructura del complejo cosechador de luz (Malis, 1999).

Efecto de diferentes fuentes de nitrógeno sobre el contenido de clorofila

La concentración de clorofila es un dato que nos permite cuantificar el crecimiento de un organismo fotoautótrofo. Existe una considerable evidencia que sustenta el hecho de que la cantidad de clorofila se correlaciona positivamente con la densidad o biomasa celular. Sin embargo algunos organismos como la Dunaliella en ciertas condiciones de cultivo acumulan metabolitos primarios o secundarios que enmascaran la presencia de clorofila en las células.
Basándonos en este experimento podemos decir que las fuentes de nitrógeno como fueron el $\mathrm{NO}_{3} \mathrm{~K}$, $\mathrm{NO}_{3} \mathrm{Na}$ y el $\mathrm{NO}_{3} \mathrm{NH}_{4}$ promovieron una acumulación de clorofila (indicador directo de crecimiento) de modo similar a lo reportado en los carotenoides totales. Pero su concentración es menor a la concentración de carotenoides totales en $\mathrm{mg} / \mathrm{l}$. Este hecho quizás esté fundamentado en que, niveles mayores de clorofila quizás promuevan una mayor producción de oxigeno, y posteriormente la producción de radicales oxidantes.

El $\mathrm{SO}_{4}\left(\mathrm{NH}_{4}\right)_{2}$, el nutriente que presentó las mas altas densidades celulares fue el que tuvo también niveles de clorofila mayores al de carotenoides a temperaturas entre 27 y $28{ }^{\circ} \mathrm{C}$. Dadas esas evidencias el autosombreado debido a altas densidades celulares evitaría probablemente la sobreproducción de oxigeno. El hecho de que medios con este nutriente en etapas estacionarias tuvieran un blanqueamiento (cambio de color verdoso anaranjado a un color blanquecino) o "bleaching" es una manifestación de un proceso fotooxidativo. Es decir el mayor nivel de clorofila en relación con el de carotenoides aunado a una disminución del $\mathrm{pH}$ pudo ser una causa de una mayor sensibilidad a procesos fotooxidativos.

Al parecer el "bleaching" por calor en Dunaliella salina se manifiesta en condiciones de estreses de corto tiempo. En los experimentos se ha observado varios bleaching cuando se realizaba repiques de los cultivos en meses en que hubo temperaturas superiores a $30{ }^{\circ} \mathrm{C}$. Estos blanqueamientos también se han observado en colonias de Dunaliella salina en placas petri. Las posibles explicaciones incluyen la variación repentina en la concentración de oxigeno y/o variación de temperaturas (Ortiz, 1990). Cuando un alga como Euglena gracilis se expone a temperaturas superiores a $33{ }^{\circ} \mathrm{C}$ inmediatamente presenta un aumento repentino de la concentración de la clorofila, que tiene su pico 15 horas después de la variación repentina de temperatura. Este incremento en la concentración de clorofila en cultivos de baja densidad celular incide en un aumento de la concentración de oxigeno que promueven procesos fotooxidativos que dañan los complejos cosechadores de luz y las membranas.

\section{Literatura citada}

Araneda P., Tapia I. \& Gómez-Silva B. 1992 a Microalgas del Norte de Chile II. Cultivos en medio de bajo costo de dos cepas de Dunaliella salina (Teodoresco, 1905) nativas del desierto de Atacama. Estud. Oceanol. 11: 5359.

Araneda P., Jimenez C. \& Gomez-Silva C. 1992 b. Microalgae fron Northern Chile III. Growth and betacarotene content of three isolates of Dunaliella salina from the Atacama desert. Rev. Biol. Mar. 27: 157-162.

Arnon D.I. 1949. Copper enzymes in isolated chloroplast polyphenyloxidase in Beta vulgaris. Plant Physiol. 24: $1-15$. 
Ben-Amotz A. \& Avron M. 1989. The biotechnology of mass culturing Dunaliella for products of commercial interest. In "Algal and Cyanobacterial Biotechnology", ed. R.C. Creswell, T. A. Rees \& N. Shah. Longman Scientific and Technical Press, Uk. : 90-114.

Borowitzka M.A. \& Borowitzka L.J. 1988. Dunaliella. In "Microalgal Biotechnology". MA Borowitzka and LJ Borowitzka (eds) Cambridge University Press, Cambridge.: 27-58.

Giordano M., Davis J. \& Bowea G. 1994. Organic carbon release by Dunaliella salina (Chlorophyta) under different growth copndition of $\mathrm{CO} 2$, nitrogen and salinity. J. Phycol. 30: 249-257.

Huppe H.C. \& Turpin D.H. 1994. Integration of carbon and nitrogen metabolism in plant and algal cells. Ann. Rev. Plant Physiol. Plant Mol. Biol. 45: 577-607.

Malis A. 1999. Photosystem-II damage and repair cycle in chloroplast: what modulates the rate of photodamage in vivo? Trends in Plant Science. 4:130-135.
Mobley H.L.T., Island M.D. \& Hausinger R.P. 1995. Microbial biology of microbial ureases. Microbiological Reviews. 59: 451-480.

Ortiz W. 1990. Protein synthesis during initial phase of the temperature -induced bleaching responses in Euglena gracilis. Plant Physiol. 93: 141-147.

Reay D.S., Nedwell D.B., Priddle J. \& Ellis-Evans J.C. 1999. Temperature dependence of inorganic uptake: Reduced affinity for nitrate at suboptimal temperatures in both algae and bacteria. Applied Environm. Microbiol. 65: 2577-2584.

Wood J.M. 1999. Osmosensing by bacteria: signals and membrane-based sensors. Microbiol. Mol. Biol. Rev. 63: 230-262.

Wycoff D.D., Davies J.P., Melies A. \& Grossman A.R. 1998. The regulation of photosynthetic electron transport during nutrient deprivation in Chlamydomonas reinhardtii Plant Physiol. 117: 129-139.

Apéndice: figura citada en el texto.
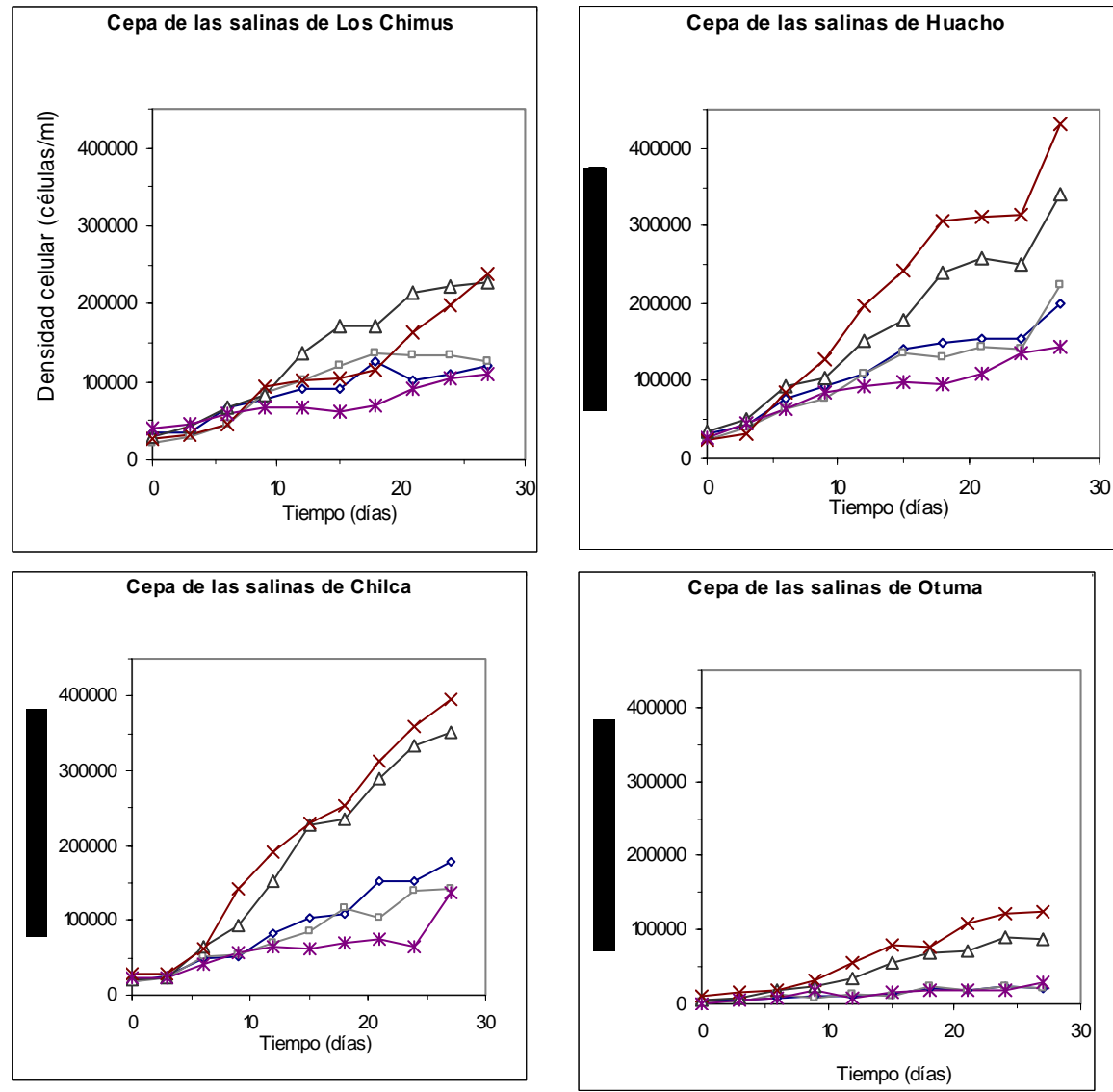

NK: $\mathrm{NO}_{3} \mathrm{~K} \quad \mathrm{NNH} 4: \mathrm{NO}_{3} \mathrm{NH}_{4}$

$\mathrm{NNa}: \mathrm{NO}_{3} \mathrm{Na} \quad \mathrm{SNH} 4: \mathrm{SO}_{4}\left(\mathrm{NH}_{4}\right)_{2}$

$\multimap$ pr/NO3K $\longrightarrow-\mathrm{pr} / \mathrm{NO}$ Na

$\triangle \mathrm{pr} / \mathrm{NO} 3 \mathrm{NH} 4 \rightarrow$ pr/SO4$(\mathrm{NH} 4) 2 \rightarrow$ pr/urea

Figura 1. Crecimiento de cuatro cepas de Dunaliella salina en medio enriquecido con diferentes fuentes de Nitrógeno.

${ }^{1}$ Laboratorio de Fisiología Vegetal de la Universidad Nacional Agraria La Molina, Lima- Perú. Apartado Postal 12-056 Lima 12 Perú. Correo electrónico: acalderon@lamolina.edu.pe 